\title{
Recent Advances of Manganese- Based Hybrid Nanomaterials for Cancer Precision Medicine
}

\author{
Xiaoman Liu ${ }^{1,2,3}$ and Pengfei Rong ${ }^{1 *}$ \\ 1 Department of Radiology, Third Xiangya Hospital, Central South University, Changsha, China, 2 Postdoctoral Research \\ Station of Clinical Medicine, Third Xiangya Hospital, Central South University, Changsha, China, ${ }^{3}$ College of Traditional \\ Chinese Medicine, Tianjin University of Traditional Chinese Medicine, Tianjin, China
}

Cancer precision medicine (CPM) could tailor the best treatment for individual cancer patients, while imaging techniques play important roles in its application. With the characteristics of noninvasion, nonionized, radiation-free, multidimensional imaging function, and real-time monitoring, magnetic resonance imaging (MRI) is an effective way

OPEN ACCESS

Edited by:

Sebastian Cerdan,

Autonomous University of

Madrid, Spain

Reviewed by:

Yunlu Dai,

University of Macau, China

Fu Wang,

X'an Jiaotong University, China

Zhen Liu,

Beijing University of Chemical

Technology, China

*Correspondence:

Pengfei Rong

rongpengfei66@163.com

Specialty section:

This article was submitted to

Cancer Imaging and

Image-directed Interventions,

a section of the journal

Frontiers in Oncology

Received: 01 July 2021

Accepted: 14 September 2021

Published: 13 October 2021

Citation:

Liu X and Rong P (2021) Recent

Advances of Manganese-Based

Hybrid Nanomaterials for

Cancer Precision Medicine.

Front. Oncol. 11:707618.

doi: 10.3389/fonc.2021.707618 for early tumor detection, and it has become a tower of strength in CPM imaging techniques. Due to linkage with nephrogenic systemic fibrosis (NSF), gadolinium (Gd)-based contrast agent (CA), which was long used in MRI, has been restricted by the Food and Drug Administration (FDA). In this review, we would like to introduce the manganese (Mn)-based CAs that could significantly increase the safety of MRI CAs by realizing more superior performance and functions simultaneously in the diagnosis and treatment of tumors. Also, recent advances in Mn-based hybrid nanomaterials for CPM are summarized and discussed.

Keywords: cancer precision medicine, magnetic resonance imaging, manganese, nanotheranostic, nanomaterials

\section{INTRODUCTION}

Cancer precision medicine (CPM), evolved with the development of novel nanoparticles (NPs) for cancer diagnosis and treatment, could tailor the best treatment for individual cancer patients. Nowadays, CPM has become popular in clinical and bioscience worldwide, with the conventionally used cancer therapies (e.g., chemotherapy, radiotherapy, and surgery) suffering from lower therapeutic efficiency and ineluctable side effects (1-4).

With a large number of nanomaterial-based new cancer therapies being emerged [e.g., photothermal therapy (PTT)/photodynamic therapy (PDT), sonodynamic therapy (SDT), magnetic hyperthermia therapy, etc.], CPM includes an extensive range of cancer management, such as cancer screening and monitoring, drug selection/prediction, and personalized immunotherapy $(2,5-8)$. CPM relies heavily on imaging methods, including computed tomography (CT), magnetic resonance imaging (MRI), positron emission tomography (PET), and optical imaging (OI), to provide distinct and precise pathological features for patients.

Owing to superb soft tissue imaging contrast, high spatial resolution, multidimensional imaging, and absence of ionizing radiation, MRI becomes increasingly available for early detection of tumors with gadolinium (Gd)-based contrast agents (CAs) most frequently used $(9,10)$. Unfortunately, Gdbased CA is in restricted use by the Food and Drug Administration (FDA) due to possibly Gd-based CA-linked medical conditions known as nephrogenic systemic fibrosis (NSF), chronic kidney 
disease (CKD), and severe complexities, which led to new concerns on the safety of Gd as MRI CAs clinically (11-14).

To increase the safety of MRI CAs, manganese (Mn) ion $\left(\mathrm{Mn}^{2+}\right)$, a non-lanthanide metal, a necessary element in cell biology, and the earliest reported CAs used for enhancing T1weighted MRI, became an optimal choice due to its paramagnetic nature, low toxicity, and high biosafety (15).

Various $\mathrm{Mn}$-based nanomaterials, such as $\mathrm{MnCl}_{2}, \mathrm{Mn}$ chelates, and $\mathrm{MnO}$ nanoparticles, have been utilized for cancer diagnosis with great biocompatibility (15-18). Multiple Mnbased nanostructures, such as nanosheets, hollows, nanocages, and nanobubbles, could act as reservoirs for efficient drug delivery (19-22). Additionally, Mn-based hybrid nanomaterials could be adaptable and responsive to both endogenous compounds in the inner tumor microenvironment (TME) (23) and external environmental stimuli, such as acidity, glutathione, temperature, $\mathrm{pH}$, enzyme, light, redox, and chemical signals. Due to those characteristics, Mn-based hybrid nanomaterials could realize demanded discharge of cargo molecular for imagingguided cancer therapy, thus minifying additional damage in normal tissues $(24,25)$.

To sum up, the paramagnetism and Fenton-like property of $\mathrm{Mn}^{2+}$ have made Mn-based hybrid nanoparticles with multiple effects, including great performance in MRI, drug delivery, and imaging-guided therapy theranostic systems to integrate diagnosis and treatment into a nanoplatform. Mn-based hybrid nanomaterials have brought a new dawn to the treatment of tumors (26).

In this review, we aimed to provide an overview of recent advances in a possible workflow of Mn-based hybrid nanomaterials used for CPM by reviewing recent emerging techniques and treatments that have been used or will be potentially used. The Mn-based hybrid nanomaterials as imaging agents, carriers for drug delivery, and theranostic agents are summarized in sections Manganese-Based Hybrid Nanomaterials as Imaging Agents, Manganese-Based Hybrid Nanomaterials as Carriers for Drug Delivery, and Manganese-Based Hybrid Nanomaterials as Theranostic Agents, respectively. We will discuss how Mn-based hybrid nanomaterials can be used as CAs for detecting and monitoring cancer progression; how they act as chemotherapeutic drug carriers to increase therapeutic index; and how they can function as theranostic agents in imaging-guided
PTT, PDT, SDT, and radiation therapy, etc. Here, we highlight the Mn-based hybrid nanomaterials as theranostic agents, and such an imaging-guided nanotheranostic platform would help to develop optimized and individualized regimens in light of patient's response and offer an opportunity to develop CPM. The progress and perspective are summarized in section Perspective.

\section{MANGANESE-BASED HYBRID NANOMATERIALS AS IMAGING AGENTS}

The noninvasive, nonionized, and radiation-free characteristics make MRI one of the most extensively utilized clinical imaging tools. However, conventional signal intensity-based MRI is still limited to its semiquantitative nature, which is susceptible to many factors. Recently, various Mn-based hybrid nanomaterials could increase T1-weighted MRI effect even in acid environment with good biocompatibility or multimodal imaging free from the effects of various conditions in the TME $(14,27,28)$. The Mnbased hybrid nanomaterials as imaging agents are summarized in Table 1, with the schematic diagram and examples of imaging effect shown in Figure 1.

T1-T2 dual-modal CAs could enable both T1 bright and T2 dark contrasts. Zhao et al. (27) prepared the multifunctional DNA-Mn-based nanoflower (DMNF), showing enhanced T1weighted MRI effect even in acid environment and high spatial resolution imaging of kidneys and liver. What is worth mentioning is that Zhou et al. (28) made a 1,4,7-triazacyclononane-N,N',N"' triacetic acid-conjugated truncated Evans blue (NEB), and after chelating with $\mathrm{Mn}(\mathrm{MnNEB})$ and bovine serum albumin (MnNEB+BSA), it could be used as novel T1-T2 dual-modal MRI CA. This study opens a new avenue for contrast-enhanced MRI diagnosis, and it also shows extraordinary promise for CPM (28).

\section{MANGANESE-BASED HYBRID NANOMATERIALS AS CARRIERS FOR DRUG DELIVERY}

Nanotechnology acts a great role in drug delivery to help revolutionize CPM. Mn-based hybrid nanomaterials, such as

TABLE 1 | Manganese-based hybrid nanomaterials as imaging agents.

\begin{tabular}{|c|c|c|c|}
\hline $\begin{array}{l}\text { Agent } \\
\text { name }\end{array}$ & Description & Tumor model & $\begin{array}{l}\text { Research group } \\
\text { and reference }\end{array}$ \\
\hline $\begin{array}{l}\text { Mn- } \\
\text { NEB } \\
+B S A\end{array}$ & $\begin{array}{l}\text { As dual-modal MRI contrast agents, Mn-NEB+BSA could greatly eliminate suspicious artifacts and false- } \\
\text { positive signals in mouse brain imaging. }\end{array}$ & $\begin{array}{l}\text { U87MG tumor-bearing } \\
\text { athymic nude mice }\end{array}$ & $\begin{array}{l}\text { Jinhao Gao and } \\
\text { Xiaoyuan Chen's } \\
\text { group (28) }\end{array}$ \\
\hline DMNF & $\begin{array}{l}\text { DMNF showed high tumor-specific MRI with enhanced T1-weighted imaging effect, which was attributed } \\
\text { to the synergistic effect of active targeting of AS1411 aptamer and acid-activated release of } \mathrm{Mn}^{2+} \\
\text { promoting the MR signal enhancement. }\end{array}$ & $\begin{array}{l}\text { MCF-7 tumor-bearing } \\
\text { BALB/c nude mice }\end{array}$ & $\begin{array}{l}\text { Dayong Yang's } \\
\text { group (27) }\end{array}$ \\
\hline HMS & $\begin{array}{l}\text { Hollow manganese silicate (HMS) nanoparticles could release } \mathrm{Mn}^{2+} \text { in physiological acidic condition as a } \\
\text { liver-specific MR contrast agent in hepatic tumor models. }\end{array}$ & $\begin{array}{l}\text { HCC, NEC, and ADC } \\
\text { tumor-bearing nude mice }\end{array}$ & $\begin{array}{l}\text { Won Jae Lee and In } \\
\text { Su Lee's group (14) }\end{array}$ \\
\hline
\end{tabular}

Mn, manganese; NEB, 1,4,7-triazacyclononane-N, N', N''-triacetic acid conjugated truncated Evans blue; BSA, bovine serum albumin; DMNF, DNA-Mn-based nanoflower; HMS, hollow manganese silicate. 


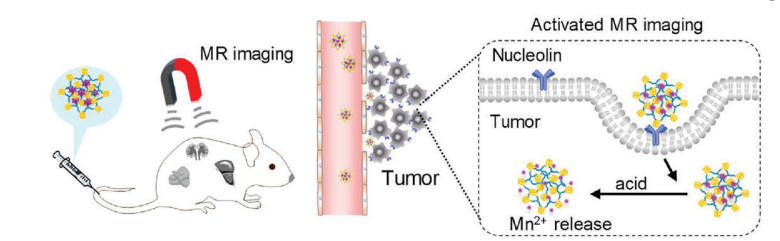

B
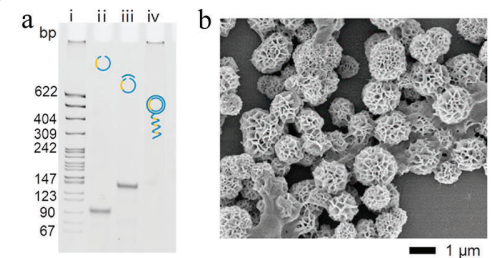

d

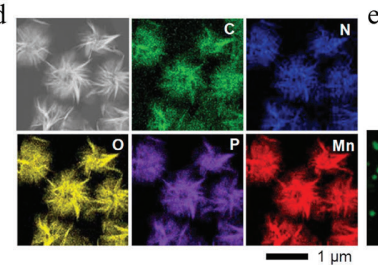

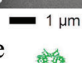

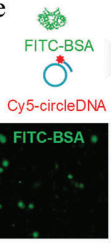

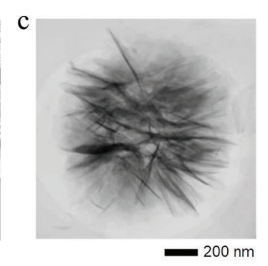

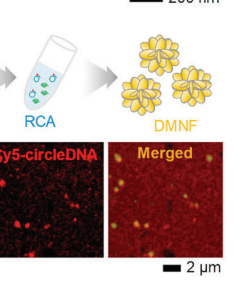

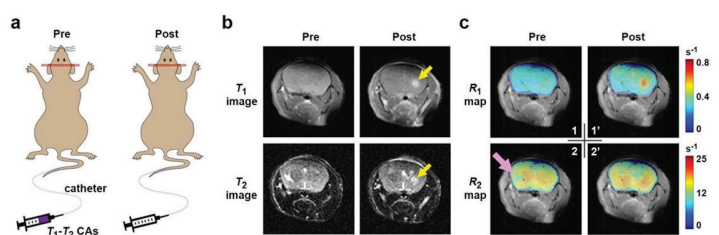

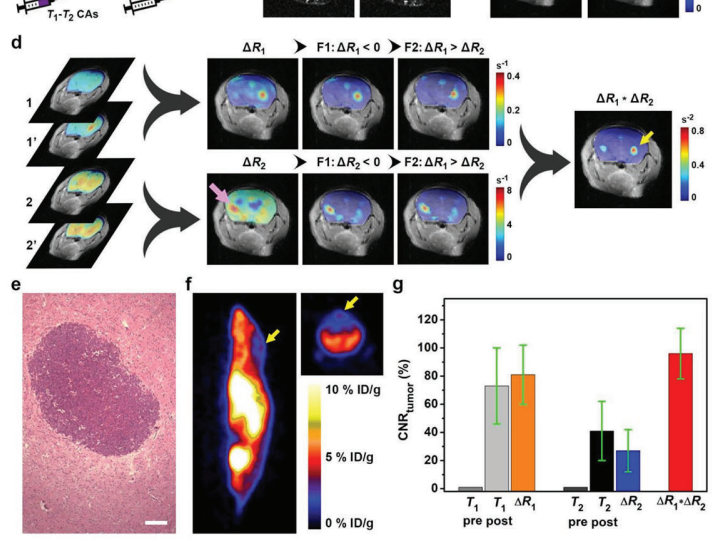

FIGURE 1 | Manganese (Mn)-based hybrid nanomaterials as imaging agents and their application in tumor. (A) Diagram of the enhanced MRI of DNA-Mn-based nanoflower (DMNF)-treated tumor-bearing mice (27). (B) Preparation and characterization of DMNF imaging agents (27). (C) Representative T1- and T2-weighted images of mouse brain at pre- and post-contrast points. T1-T2 dual-modal MRI in brain tumor model through the synthesized MRI contrast agents, NOTA conjugated NEB chelating with $\mathrm{Mn}^{2+}$ (Mn-NEB) and BSA (Mn-NEB+BSA) (28). DMNF, DNA-Mn-based nanoflower; NOTA, N, N', N"'-triacetic acid.

nanosheets, hollow mesoporous nanoshells, and nanocubes, have a high surface-to-volume ratio fit for drug delivery and could produce $\mathrm{Mn}^{2+}$ for MRI (20). Currently fabricated composite nanoparticles used for drug delivery include the nanoparticle for the carrier and chemotherapeutic drug for cancer \{e.g., doxorubicin [DOX], paclitaxel [PTX], methotrexate [MTX], arsenic trioxide [ATO], cisplatin [cis-diamminedichloroplatinum (CDDP)], etc.\} or non-tumor-specific drugs (e.g., hydroxychloroquine, verteporfin, 5-fluorouracil, osteopontin siRNA, etc.) that is either adsorbed, dissolved, or dispersed throughout the nanoparticle complex or covalently attached to the surface of nanoparticles (5). Also, they hold great potential to simultaneously codeliver more drugs in combination therapy. The delivery of non-cytotoxic prodrugs to cancer cells is one of the newer applications (29).

Furthermore, drugs can be formulated at a nanoscale level to increase its therapeutic efficiency. Nanoscale drug delivery systems (nano-DDSs) have already been proposed as a promising way to realize tumor-specific treatment by being adaptable and responsive to many endogenous substances and external stimuli, such as acidity, overexpressed hydrogen peroxide (23), pH, enzyme, light, temperature, and magnetic field.

Hence, numerous smart hybrid nanomaterials with one or dual stimuli-responsive (e.g., lower $\mathrm{pH}$, hypoxia, tumor-specific enzymes such as glutathione, etc.) drug-releasing and one or dual-mode diagnostic imaging functions (particularly MRI) have been developed to realize improved therapeutic specificity and efficacy $(12,13,19,21,22,24,25,30-48)$. The Mn-based hybrid nanomaterials as carriers for drug delivery are summarized in Table 2, with examples of the schematic diagram for drug delivery system, characterization analysis, and curative effect shown in Figure 2. It is worth noting that redox-sensitive Mn-SS (disulfide)/DOX@PDA (polydopamine)-PEG polymers (NCPs) designed by Zhao et al. (30) served as a T1 CA under MRI and showed a glutathione (GSH)-responsive release of DOX. Huang et al. (9) fabricated theranostic nanocomposites $\mathrm{Mn}$ porphyrin\& $\mathrm{Fe}_{3} \mathrm{O}_{4} @ \mathrm{SiO}_{2} @ \mathrm{PAA}-\mathrm{ARGD}$ and effectively used them in T1- and T2-weighted MRI and $\mathrm{pH}$-responsive drug release. Wang et al. (49) reported the one-pot synthesis of biocompatible arginine-rich Mn silicate nanobubbles (AMSNs) with high tumor killing activity via the glutathione-dependent peroxidases 4 (GPX4)-mediated ferroptosis pathway. Such imaging-guided drug-carrying platforms would therefore tremendously promote the development of CPM.

\section{MANGANESE-BASED HYBRID NANOMATERIALS AS THERANOSTIC AGENTS}

Many efforts have been made for cancer therapy, and the idea of theranostics could help develop a smart nanoparticle to integrate cancer diagnosis, drug delivery, and therapy monitoring simultaneously in a system (50). The intelligent stimuliresponsive manner could offer an efficient strategy for CPM by employing the unique features of TME or clinical external irradiations. With the improvement of polymerization and emulsifying techniques, nanoparticles could be made with hydrophilic and hydrophobic facets to load with different 
TABLE 2 | Manganese-based hybrid nanomaterials as carriers for drug delivery.

\begin{tabular}{|c|c|c|c|c|}
\hline $\begin{array}{l}\text { Delivered } \\
\text { molecules }\end{array}$ & Agent name & Description & Tumor model & $\begin{array}{l}\text { Research group and ref- } \\
\text { erence }\end{array}$ \\
\hline DOX & $\begin{array}{l}\mathrm{HMnO}_{2} \\
\text { nanoshells }\end{array}$ & $\begin{array}{l}\text { Hollow mesoporous } \mathrm{MnO}_{2}\left(\mathrm{HMnO}_{2}\right) \text { nanoshells with DOX loaded could be used for } \\
\text { tumor-specific therapy in pH-responsive MRI. }\end{array}$ & $\begin{array}{l}4 \mathrm{~T} 1 \text { tumor-bearing } \\
\text { Balb/c mice }\end{array}$ & Zhuang Liu's group (24) \\
\hline DOX & $\begin{array}{l}\text { Mn-SS/ } \\
\text { DOX@PDA- } \\
\text { PEG NCPS }\end{array}$ & $\begin{array}{l}\text { Redox-sensitive Mn-SS (disulfide)/DOX@PDA (polydopamine)-PEG polymers (NCPs) } \\
\text { for T1-contrast MRI and glutathione (GSH)-responsive release of DOX }\end{array}$ & $\begin{array}{l}4 \mathrm{~T} 1 \text { tumor-bearing } \\
\text { mice }\end{array}$ & $\begin{array}{l}\text { Zili Ge and Zhuang Liu's } \\
\text { group (30) }\end{array}$ \\
\hline DOX & $\begin{array}{l}\mathrm{MnO}_{2} \text {-PEG- } \\
\text { FAVDOX } \\
\text { nanosheets }\end{array}$ & $\begin{array}{l}\text { A redox/pH dual responsive nanotheranostic platform, } \mathrm{MnO}_{2}-\mathrm{PEG}-\mathrm{FA} / \mathrm{DOX} \\
\text { nanosheets through } \mathrm{MnO}_{2} \text { nanosheets combined with } \mathrm{FA} \text { and DOX for MRI and } \\
\text { chemotherapy }\end{array}$ & $\begin{array}{l}\text { S180 tumor-nearing } \\
\text { mice }\end{array}$ & $\begin{array}{l}\text { Zhenzhong Zhang and Yun } \\
\text { Zhang's group (35) }\end{array}$ \\
\hline DOX & $\begin{array}{l}\text { DOX-GOX- } \\
\text { MnCaP NPs }\end{array}$ & $\begin{array}{l}\text { A pH-responsive DOX-loaded glucose oxidase (GOx) with MnCaP spherical } \\
\text { nanoparticles for MRI and cascade reaction-enhanced cooperative cancer treatment }\end{array}$ & $\begin{array}{l}\text { 4T1 tumor-bearing } \\
\text { mice }\end{array}$ & Peng Huang's group (39) \\
\hline DOX & BMDN & $\begin{array}{l}\mathrm{MnO}_{2} / \mathrm{DOX} \text {-loaded albumin nanoparticles (BMDN) for MRI and simultaneous } \\
\text { chemotherapy }\end{array}$ & $\begin{array}{l}\text { MCF-7/ADR tumor- } \\
\text { bearing mice }\end{array}$ & $\begin{array}{l}\text { Huabing Chen and Hu-Lin } \\
\text { Jiang's group (31) }\end{array}$ \\
\hline DOX & USMO@MSNs & USMO@MSNs loading DOX for pH-switching MRI and chemotherapy & $\begin{array}{l}\text { HSC3 tumor- } \\
\text { bearing nude mice }\end{array}$ & $\begin{array}{l}\text { Renfei Wang and Duohong } \\
\text { Zou (34) }\end{array}$ \\
\hline MTX & $\begin{array}{l}\text { MTX- } \\
\text { Mn@PEG } \\
\text { NCPs }\end{array}$ & $\begin{array}{l}\text { A chelating agent free, stoichiometry, and pH-responsive NCPs for MRI-guided MTX } \\
\text { delivery }\end{array}$ & $\begin{array}{l}\text { HeLa tumor-nearing } \\
\text { BALB/c nude mice }\end{array}$ & $\begin{array}{l}\text { Youfu Wang, Dawei Li and } \\
\text { Xinyuan Zhu's group (38) }\end{array}$ \\
\hline ATO & $\begin{array}{l}{\left[\mathrm{Mn}\left(\mathrm{HAsO}_{3}\right)\right]} \\
\mathrm{n} @ \mathrm{SiO}_{2}\end{array}$ & 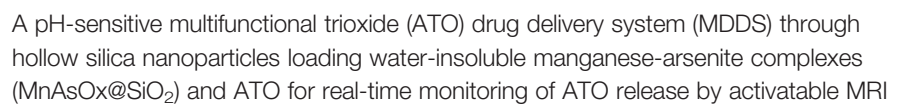 & $\begin{array}{l}\text { H22 tumor-nearing } \\
\text { BALB/c mice }\end{array}$ & Jinhao Gao's group (36) \\
\hline CDDP & $\begin{array}{l}\mathrm{MnO}_{2} / \mathrm{HA} \\
\text { CDDP } \\
\text { nanosheets }\end{array}$ & $\begin{array}{l}\mathrm{MnO}_{2} / \mathrm{HA} / \mathrm{CDDP} \text { nanosheets }\left(\mathrm{MnO}_{2} \text { nanosheets functionalized by } \mathrm{HA} \text {, with CDDP }\right. \\
\text { absorbed) for pH-responsive } \mathrm{MRI} \text { and delivering CDDP }\end{array}$ & $\begin{array}{l}\text { A549 tumor-bearing } \\
\text { mice }\end{array}$ & $\begin{array}{l}\text { Zhenzhong Zhang and Yun } \\
\text { Zhang's group (37) }\end{array}$ \\
\hline $\mathrm{HCQ}$ & $\begin{array}{l}\mathrm{HA}-\mathrm{Mn}_{2} \mathrm{O}_{3} / \\
\mathrm{HCQ}\end{array}$ & $\begin{array}{l}\text { TME-responsive drug release and tumor targeting drug carriers-Hollow mesoporous } \\
\mathrm{Mn}_{2} \mathrm{O}_{3} \mathrm{NPs} \text { conjugated with hyaluronic acid }(\mathrm{HA}) \text { loading hydroxychloroquine }(\mathrm{HCQ} \text {, } \\
\text { traditional autophagy inhibitor) into the hollow core, for MRI-guided in situ autophagy } \\
\text { inhibition }\end{array}$ & $\begin{array}{l}4 \mathrm{~T} 1 \text { tumor-bearing } \\
\mathrm{BALB} / \mathrm{c} \text { mice }\end{array}$ & $\begin{array}{l}\text { Lin Hou and Zhenzhong } \\
\text { Zhang's group (21) }\end{array}$ \\
\hline
\end{tabular}

$\mathrm{HMnO}_{2}$, hollow mesoporous $\mathrm{MnO}_{2}$; DOX, doxorubicin; MRI, magnetic resonance imaging; PDA, polydopamine; SS, disulfide; GSH, glutathione; GOx, glucose oxidase; MnCaP, manganese-doped calcium phosphate; BMDN, BSA-MnO ${ }_{2}$-DOX nanoparticles; USMO@MSNs, Ultrasmall manganese oxide-capped mesoporous silica nanoparticles; MCO-70 NPS, Hollow manganese/cobalt oxide nanoparticles with an average size of $70 \mathrm{~nm}$; MTX, methotrexate; MDDS, multifunctional drug delivery system; MnAsOx@SiO 2 , hollow silica nanoparticles loading water-insoluble manganese-arsenite complexes; $H A$, hyaluronic acid; CDDP, cis diamminedichloroplatinum; TME, tumor microenvironment; $H A$, hyaluronic acid; $H C Q$, hydroxychloroquine; $\mathrm{BPD}$, benzoporphyrin derivative; $\mathrm{Mn}_{2} \mathrm{O}_{3}$, manganese trioxide; OPN, osteopontin; ZIF, zeolitic imidazolate framework.

active materials for theranostics. The Mn-based hybrid nanomaterials as imaging agents and carriers for drug delivery have been summarized and discussed in this section, and the Mn-based hybrid nanomaterials as theranostic agents are summarized in Table 3, with the schematic diagram and examples shown in Figure 3.

\section{Imaging-Guided Photothermal Therapy}

PTT, a combination of photothermal nanomaterials and light irradiation, becomes a clinically promising modality for cancers. It could controllably and selectively heat the target area to minimize thermal damage.

Many Mn-based hybrid nanomaterials used for imagingguided PTT have been developed $(11,48,71,73-77)$, such as nanopetals of $\mathrm{Mn}_{3} \mathrm{O}_{4}$ hybrid nanomaterials for multifunctional imaging-guided PTT (51), a 2-D nanoplatform based on $\mathrm{Cu}_{2} \mathrm{MnS}_{2}$ nanoplates for $\mathrm{MRI} /$ multispectral optoacoustic tomography (MSOT) dual-modal imaging-guided PTT $(52,78)$, a plasmonic modulation strategy of Gold Nanorods (GNRs) through $\mathrm{MnO}_{2}$ coating for TME-responsive photoacoustic (PA)/MR duplex imaging guided NIR-II PTT (67), and a gold@ $\mathrm{MnO}_{2}$ (Au@ $\mathrm{MnO}_{2}$ ) core-shell nanostructure as a GSH-triggered smart theranostic agent for PA and MR dual imaging-guided PTT $(53,68)$.

\section{Imaging-Guided Photodynamic Therapy}

PDT has emerged as a promising therapeutic option for cancers, and it could generate cytotoxic oxygen-based 

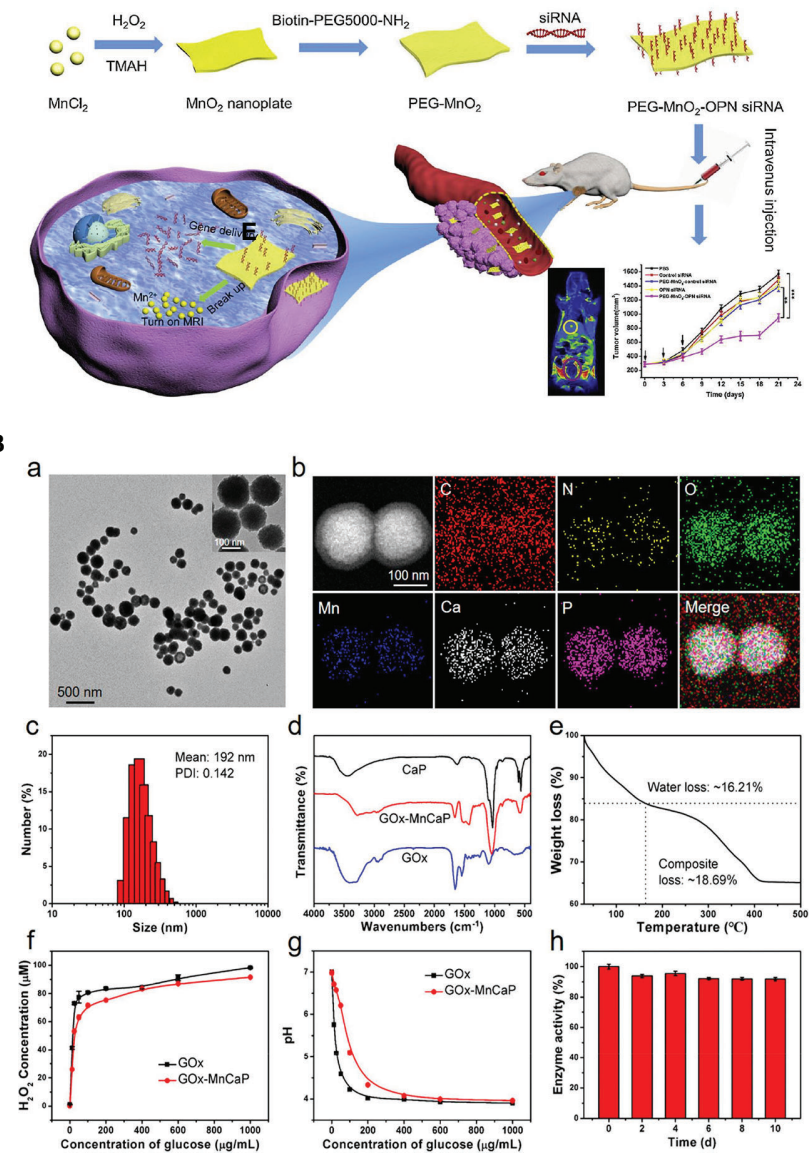
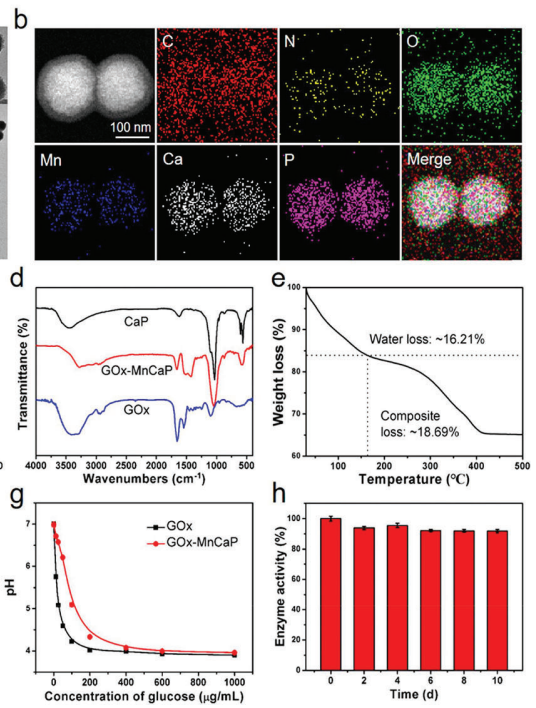

C

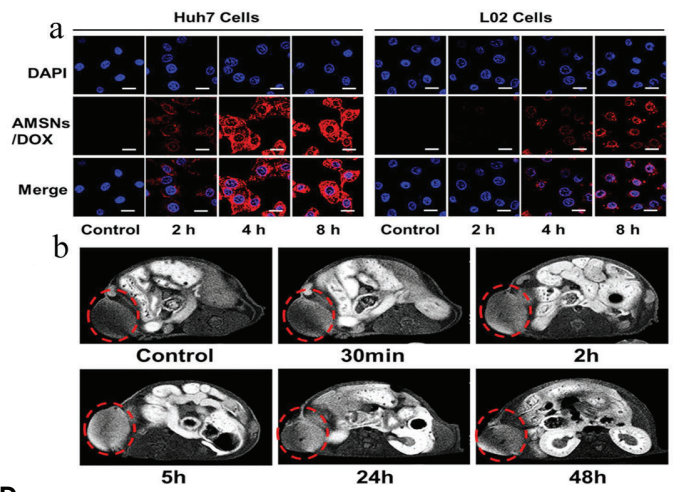

D
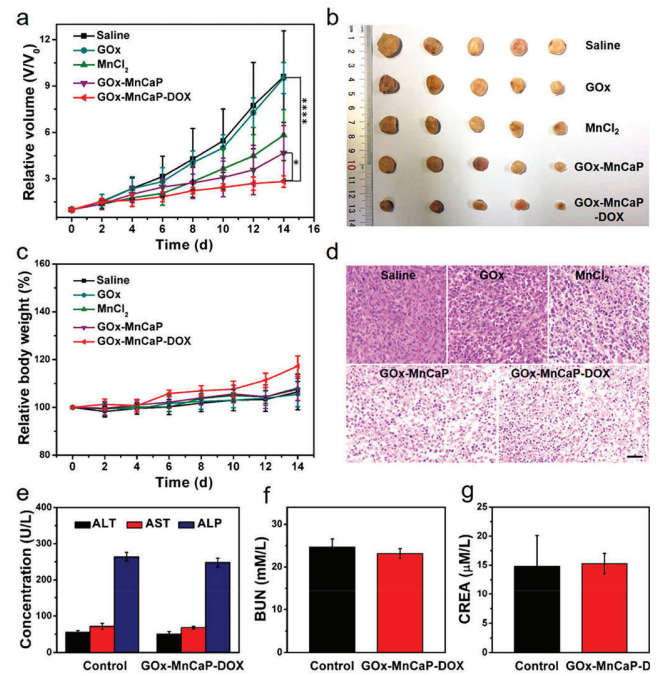

FIGURE 2 | Mn-based hybrid nanomaterials as carriers for drug delivery and their application in tumor. (A) Schematic illustration of PEG-MnO 2 -OPN siRNA (20). (B) Preparation and characterization of GOx-MnCaP-DOX, glucose oxidase (GOx) with manganese-doped calcium phosphate (MnCaP), and doxorubicin (DOX) (39). (C) Tumor cell-selective uptake analysis by confocal laser scanning microscope and in vivo tumor homing behavior evaluation by T1-weighted MRI of arginine-rich manganese silicate nanobubbles loading DOX (AMSNs/DOX) (49). (D) The in vivo antitumor efficacy of GOx-MnCaP-DOX on the 4T1 tumor-bearing mouse model (39). OPN, Osteopontin; GOx, Glucose; MnCaP, manganese-doped calcium phosphate; DOX, doxorubicin; AMSNs, Arginine-rich manganese silicate nanobulles.

molecular species via photosensitizer to ablate tumor growth by inducing cell apoptosis, necrosis, or autophagy. As a new noninvasive modality, PDT could enhance the conventional cancer treatment by overcoming drug resistance or escape pathways.

A lot of Mn-based hybrid nanoparticles were synthesized for imaging-guided PDT diagnosis and treatment (10, 54, 55, 79, 80). For example, Zhang et al. (10) have proven that Mn-doped iron oxide nanoparticles modified with denatured BSA (MnIOdBSA) and Fmoc-L-L/Mn ${ }^{2+} / \mathrm{Ce} 6$ nanoparticles (FMCNPs) could improve antitumor PDT efficacy. Also, oxygen-generating theranostic nanoparticles (CDM NPs) with $\mathrm{MnO}_{2}$ could be applied for trimodal imaging-guided combined PDT in breast cancer (69). A multifunctional DNA-templated silver nanoclusters/porphyrin/ $\mathrm{MnO}_{2}$ nanoplatform could be used for non-labeled fluorescence images of $\mathrm{Zn}^{2+}$ and 635-nm red lighttriggered PDT (56). The $\mathrm{MnO}_{2}$ NP-based PDT nanocomplex could generate oxygen to overcome the limitation of insufficient oxygen level in tumors (55).

\section{Imaging-Guided Sonodynamic Therapy}

SDT is an alternative promising method for cancers by generating reactive oxygen species (ROS), ROS to induce cell death with low-intensity ultrasound irradiation combined with nontoxic sonosensitizers $(81,82)$. It is characterized by high therapeutic efficiency with the advantages of noninvasiveness and mitigated side effects.

Mn-based theranostic agents could integrate imaging and therapy into a single nano-platform for imaging-guided SDT. It has been reported that even in the presence of skull, sinoporphyrin sodium (DVDMS) chelating with Mn (DVDMS-Mn-LPs) could effectively inhibit the tumor growth (57). The efficacy of SDT could be severely inhibited by hypoxia and high glutathione in TME, while a Mn porphyrin-based metal-organic framework 
TABLE 3 | Manganese-based hybrid nanomaterials as theranostic agents.

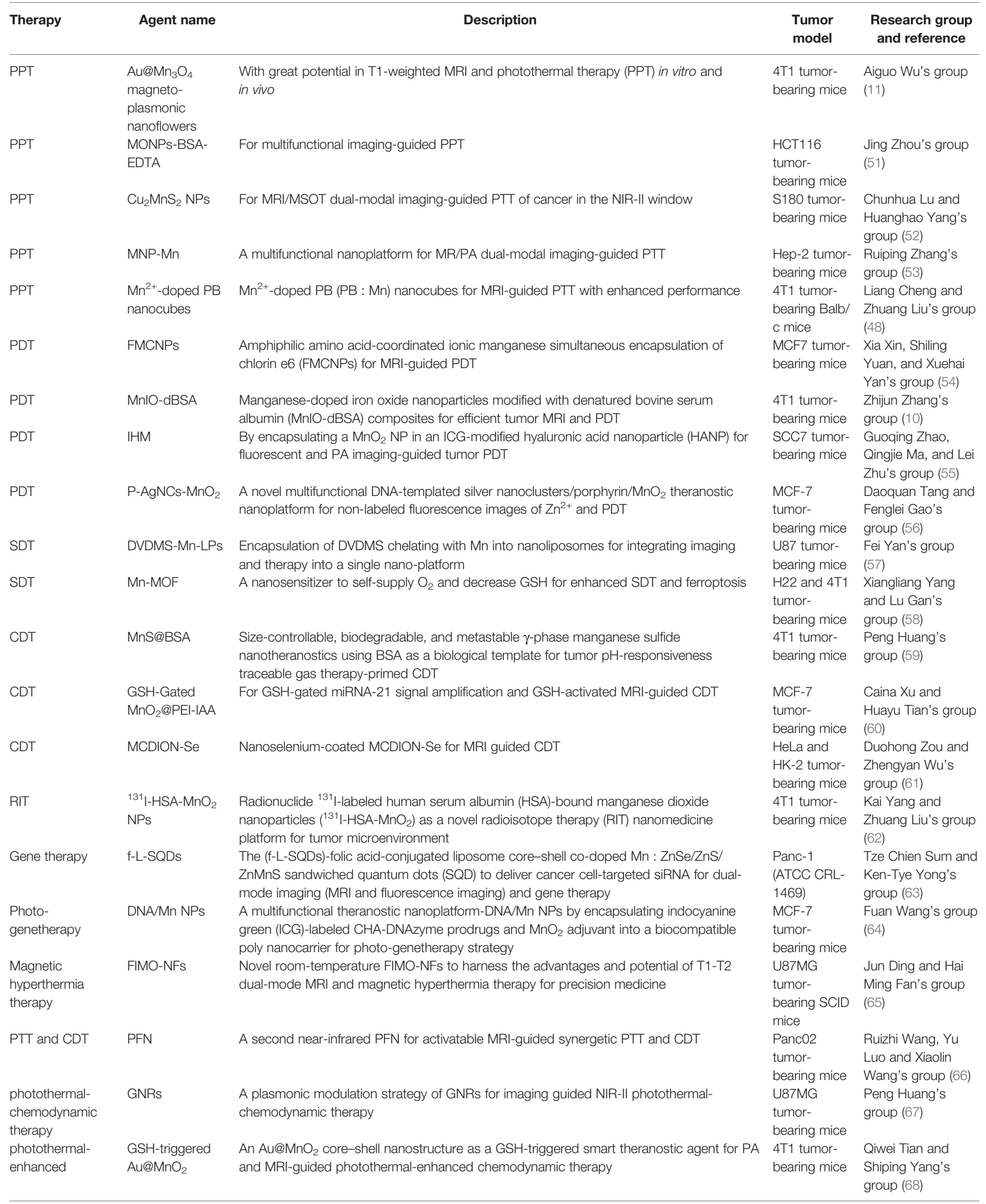


TABLE 3 | Continued

\begin{tabular}{|c|c|c|c|c|}
\hline Therapy & Agent name & Description & $\begin{array}{l}\text { Tumor } \\
\text { model }\end{array}$ & $\begin{array}{l}\text { Research grou } \\
\text { and reference }\end{array}$ \\
\hline \multicolumn{5}{|l|}{$\begin{array}{l}\text { chemodynamic } \\
\text { therapy }\end{array}$} \\
\hline $\begin{array}{l}\text { chemo- } \\
\text { photodynamic } \\
\text { therapy }\end{array}$ & CDM NPs & $\begin{array}{l}\text { Oxygen-generating theranostic nanoparticles by hierarchically assembling DOX, Ce6, and } \\
\mathrm{MnO}_{2} \text { with poly-b-poly-b-poly for trimodal imaging-guided combined chemo- } \\
\text { photodynamic therapy }\end{array}$ & $\begin{array}{l}\text { MCF-7 } \\
\text { tumor- } \\
\text { bearing mice }\end{array}$ & $\begin{array}{l}\text { ZhiYong Qian's } \\
\text { group (69) }\end{array}$ \\
\hline
\end{tabular}

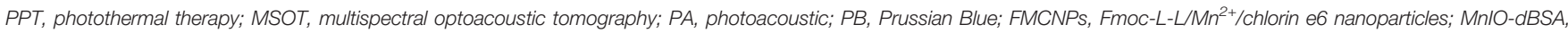
manganese doped iron oxide nanoparticles modified with denatured bovine serum albumin; HANP, hyaluronic acid nanoparticle; PDT, photodynamic therapy; SDT, sonodynamic therapy; DVDMS, organic sinoporphyrin sodium; CDT, chemodynamic therapy; MCDION-Se, nanoselenium coated manganese carbonate-deposited iron oxide nanoparticle; HSA, human serum albumin; RIT, radioisotope therapy; SQD, sandwiched quantum dots; ICG, indocyanine green; CHA, catalytic hairpin assembly; FIMO-NFs, ferromagnetic IMO nanoflowers; PFN, photothermal Fenton nanocatalyst; GNRs, gold nanorods; CDM NPs, chlorin e6-DOX-MnO 2 nanoparticles.

A

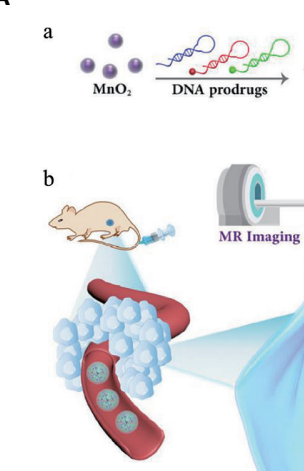

B

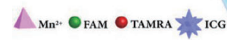

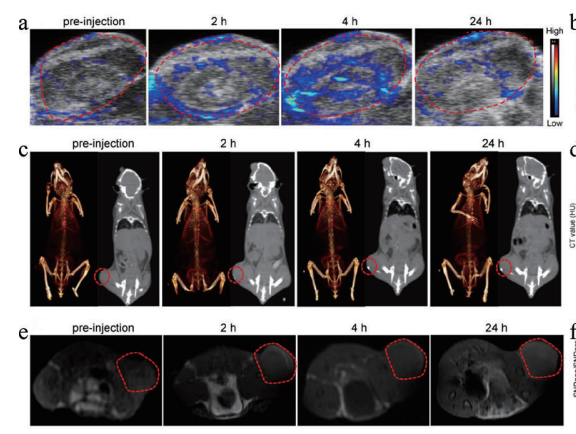

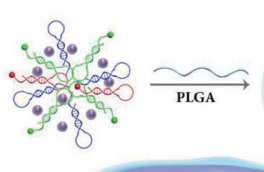
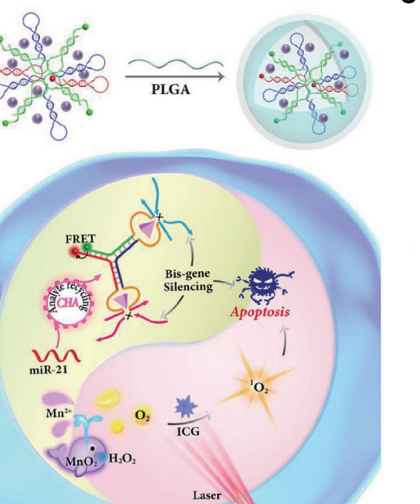

Laser
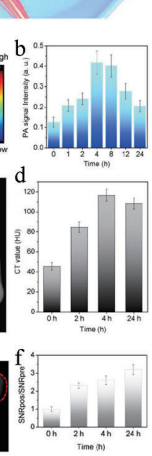

C
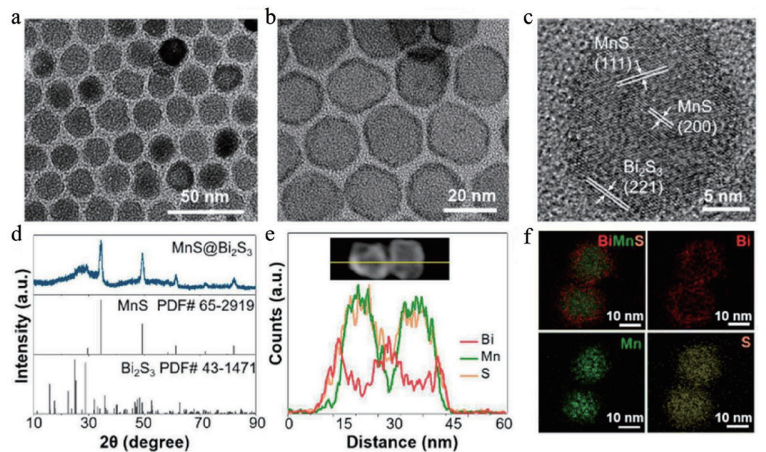

D

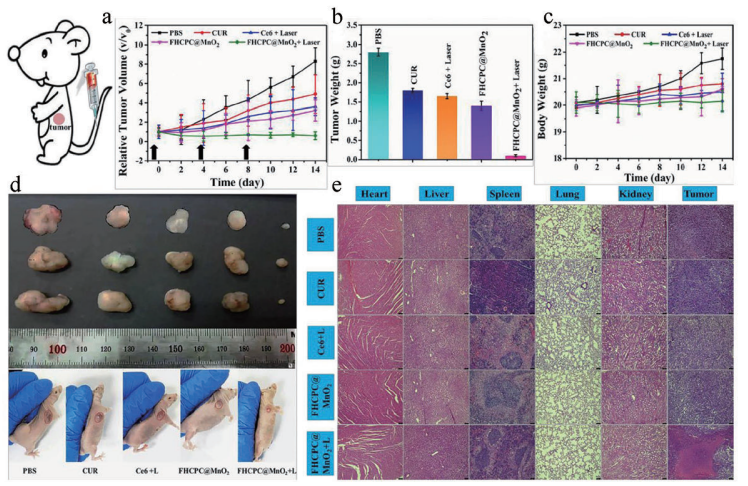

FIGURE 3 | Manganese (Mn)-based hybrid nanomaterials as theranostic agents and their application in tumor. (A) Schematic illustration of multifunctional

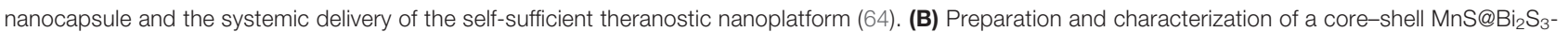
PEG nanostructure theranostic agents (70). (C) In vivo multimodal imaging (PA images, CT images, and MR images with the red circles mark the tumors) and corresponding signal analysis of tumor-bearing mice before and after intravenous injection of the monolayer bi-anchored Mn boride nanosheets (MBBN) (71). (D) In vivo therapeutic evaluation of $\mathrm{FHCPC} @ \mathrm{MnO}_{2}$ nanoflowers (polyphosphazene coated onto $\mathrm{Fe}_{3} \mathrm{O}_{4}$ nanoclusters, with $\mathrm{MnO}_{2}$ nanosheets as outer shell). Scale bar $=75 \mu \mathrm{m}$ (72). PA, photoacoustic; FHCPC, coating multifunctional polyphosphazenes onto $\mathrm{Fe}_{3} \mathrm{O}_{4}$ nanoclusters and then growing manganese oxide nanosheets as outer shell; MBBN, Bi-anchored manganese boride nanosheets.

(Mn-MOF) could improve antitumor immunity and immunosuppressive microenvironment upon ultrasound irradiation to show great potential for hypoxic cancer therapy (58).

\section{Other Imaging-Guided Therapies}

Mn-based hybrid nanomaterials also hold great potential for many other traceable therapies for cancer, such as chemodynamic therapy (CDT) $(60,61)$, radiation therapy $(83)$, magnetic hyperthermia therapy, and combination therapy (70, 84, 85).

For $\mathrm{pH}$-responsive traceable gas therapy-primed CDT, a $\gamma$ phase Mn sulfide nanotheranostics using bovine serum albumin (MnS@BSA) could greatly suppress tumor growth (59). During radiation therapy, ionizing radiation will damage both normal 
tissues and tumors (86), and hypoxia within TME would often lead to the resistance to radiotherapy. To improve the effect of radiation therapy, radionuclide ${ }^{131} \mathrm{I}$-labeled human serum albumin (HSA)-bound $\mathrm{MnO}_{2}$ nanoparticles ( ${ }^{131} \mathrm{I}-\mathrm{HSA}-\mathrm{MnO}_{2}$ ) could function as an effective agent to show great efficacy in tumor treatment (62). The novel room-temperature ferromagnetic wüstite iron-manganese oxide nanoflowers (FIMO-NFs) could harness the advantages and potential of dual-mode MRI and magnetic hyperthermia therapy to induce cancer cell apoptosis (65).

$\mathrm{Mn}^{2+}$-doped bio-response theranostic NP could be designed for tumor-specific enhanced combination therapy under the guidance of multimodal imaging $(64,66,87,88)$. Pd@Au bimetallic NP-decorated hollow mesoporous $\mathrm{MnO}_{2}\left(\mathrm{H}-\mathrm{MnO}_{2}\right)$ NPs could achieve both nucleus-targeted PTT and TME hypoxia relief-enhanced PDT (89). As an intelligent nanoflower composite with multistage $\mathrm{H}_{2} \mathrm{O}_{2} / \mathrm{pH} / \mathrm{GSH}$-responsive properties, FHCPC@ $\mathrm{MnO}_{2}$ could realize the specific release of drugs in tumor and significantly increase the synergetic therapeutic effect (72).

\section{PERSPECTIVE}

Cancer still remains a significant challenge worldwide, and the new discovered theranostic nanomaterials, such as Mn-based hybrid nanomaterials, which make diagnosis and treatment together in a unified platform, provide a novel therapy specialized for tumors. Since nanomaterials for theranostics create great new opportunities in developing CPM, this review focused on Mn-based nanoparticles with various applications (used as imaging agents, drug delivery, and theranostic agents) in CPM. Although a multitude of Mn-based hybrid nanomaterials

\section{REFERENCES}

1. Cheng T, Liu J, Ren J, Huang F, Ou H, Ding Y, et al. Green Tea CatechinBased Complex Micelles Combined With Doxorubicin to Overcome Cardiotoxicity and Multidrug Resistance. Theranostics (2016) 6(9):1277-92. doi: $10.7150 /$ thno. 15133

2. Deng X, Nakamura Y. Cancer Precision Medicine: From Cancer Screening to Drug Selection and Personalized Immunotherapy. Trends Pharmacol Sci (2017) 38(1):15-24. doi: 10.1016/j.tips.2016.10.013

3. Jameson JL, Longo L. Precision Medicine Is 'Personalized, Problematic, and Promising'. N Engl J Med (2015) 372(23):2229-34. doi: 10.1056/ NEJMsb1503104

4. Adir O, Poley M, Chen G, Froim S, Krinsky N, Shklover J, et al. Integrating Artificial Intelligence and Nanotechnology for Precision Cancer Medicine. Adv Mater (Deerfield Beach Fla) (2020) 32(13):e1901989. doi: 10.1002/ adma.201901989

5. Avnesh S, Thakor SSG. Nanooncology: the Future of Cancer Diagnosis and Therapy. CA Cancer J Clin (2013) 63(6):395-418. doi: 10.3322/caac.21199

6. Wang X, Yang L, Chen ZG, Shin DM. Application of Nanotechnology in Cancer Therapy and Imaging. CA Cancer J Clin (2008) 58(2):97-110. doi: 10.3322/CA.2007.0003

7. Yang B, Lin H, Dai C, Chen Y, Shi J. "Stepwise Extraction" Strategy-Based Injectable Bioresponsive Composite Implant for Cancer Theranostics. Biomaterials (2018) 166:38-51. doi: 10.1016/j.biomaterials.2018.03.010

8. Joo J, Choi M, Jang S, Choi S, Park S, Shin D, et al. Realizing Cancer Precision Medicine by Integrating Systems Biology and Nanomaterial Engineering. $A d v$ have not been successfully used in the clinic, several welldesigned Mn-based hybrid nanoparticles provide a new promising treatment option in the near future. What is worth emphasizing is that the novel nanoparticles should be thoroughly characterized, whether used as imaging agents, carriers for drugs, or theranostic platforms, and the toxicity studies in both cell culture and animal models are needed before they can be applied clinically. A future perspective is proposed for further research and development of complex targeted, multistage responsive nanomedical drug delivery systems with high intelligence, precision, and minimum toxicity for personalized cancer diagnosis and effective therapy. A major obstacle in designing theranostic Mn-based hybrid nanomaterials might be that providing target specificity to biomaterials for enhancing therapeutic effect and visualization in CPM. With the aid of multimode imaging, theranostic nanoparticles can visualize and monitor drug delivery and therapeutic responses at tumor site.

\section{AUTHOR CONTRIBUTIONS}

XL and PR contributed to the conception, design, writing, and final approval of the article. All authors contributed to the article and approved the submitted version.

\section{FUNDING}

This work was supported by the National Natural Science Foundation of China $(81771827,82071986)$ and the 65th Batch of China Postdoctoral Science Foundation (2019M652806).
Mater (Deerfield Beach Fla) (2020) 32(35):e1906783. doi: 10.1002/ adma.201906783

9. Huang X, Yuan Y, Ruan W, Liu L, Liu M, Chen S, et al. pH-Responsive Theranostic Nanocomposites as Synergistically Enhancing Positive and Negative Magnetic Resonance Imaging Contrast Agents. J Nanobiotechnol (2018) 16(1):30. doi: 10.1186/s12951-018-0350-5

10. Zhang M, Cao Y, Wang L, Ma Y, Tu X, Zhang Z. Manganese Doped Iron Oxide Theranostic Nanoparticles for Combined T1 Magnetic Resonance Imaging and Photothermal Therapy. ACS Appl Mater Interfaces (2015) 7 (8):4650-8. doi: 10.1021/am5080453

11. Ijaz Dar G, Iqbal MZ, Akakuru OU, Yao C, Awiaz G, Wu A. Facile Synthesis of Au@Mn(3)O(4) Magneto-Plasmonic Nanoflowers for T(1)-Weighted Magnetic Resonance Imaging and Photothermal Therapy of Cancer. J Mater Chem B (2020) 8(36):8356-67. doi: 10.1039/d0tb01526a

12. Karimi Shervedani R, Samiei Foroushani M, Kefayat A, Torabi M, Rahnemaye Rahsepar F. Construction and Characterization of a Theranostic System Based on Graphene/Manganese Chelate. Biosens Bioelectron (2018) 117:794-801. doi: 10.1016/j.bios.2018.07.011

13. Zuo H, Chen W, Li B, Xu K, Cooper H, Gu Z, et al. MnAl Layered Double Hydroxide Nanoparticles as a Dual-Functional Platform for Magnetic Resonance Imaging and siRNA Delivery. Chem (Weinheim an der Bergstrasse Germany) (2017) 23(57):14299-306. doi: 10.1002/chem.201702835

14. Kim J, Jang M, Kumari N, Choi J, Im G, Kwon T, et al. Differential Characterization of Hepatic Tumors in MR Imaging by Burst-Released MnIons From Hollow Manganese-Silicate Nanoparticles in the Liver. Biomaterials (2020) 230:119600. doi: 10.1016/j.biomaterials.2019.119600 
15. Loving GS, Mukherjee S, Caravan P. Redox-Activated Manganese-Based MR Contrast Agent. J Am Chem Soc (2013) 135(12):4620-3. doi: 10.1021/ ja312610j

16. Kim KH, Fonda JR, Lawler EV, Gagnon D, Kaufman JS. Change in Use of Gadolinium-Enhanced Magnetic Resonance Studies in Kidney Disease Patients After US Food and Drug Administration Warnings: A CrossSectional Study of Veterans Affairs Health Care System Data From 20052008. Am J Kidney Dis (2010) 56(3):458-67. doi: 10.1053/j.ajkd.2010.03.027

17. Milatovic D, Zaja-Milatovic S, Gupta RC, Yu Y, Aschner M. Oxidative Damage and Neurodegeneration in Manganese-Induced Neurotoxicity. Toxicol Appl Pharmacol (2009) 240(2):219-25. doi: 10.1016/j.taap.2009.07.004

18. Winslow JWW, Limesand KH, Zhao N. The Functions of ZIP8, ZIP14, and $\mathrm{ZnT} 10$ in the Regulation of Systemic Manganese Homeostasis. Int J Mol Sci (2020) 21(9):1-15. doi: 10.3390/ijms21093304

19. Wang Y, Shang W, Zhong H, Luo T, Niu M, Xu K, et al. Tumor Vessel Targeted Self-Assemble Nanoparticles for Amplification and Prediction of the Embolization Effect in Hepatocellular Carcinoma. ACS Nano (2020) 14 (11):14907-18. doi: 10.1021/acsnano.0c04341

20. Shi M, Wang S, Zheng S, Hou P, Dong L, He M, et al. Activatable MRIMonitoring Gene Delivery for the Theranostic of Renal Carcinoma. Colloids Surf B Biointerfaces (2020) 185:110625. doi: 10.1016/j.colsurfb.2019.110625

21. Zhang H, Ren Y, Cao F, Chen J, Chen C, Chang J, et al. In Situ Autophagy Disruption Generator for Cancer Theranostics. ACS Appl Mater Interfaces (2019) 11(33):29641-54. doi: 10.1021/acsami.9b10578

22. Ren Q, Yang K, Zou R, Wan Z, Shen Z, Wu G, et al. Biodegradable Hollow Manganese/Cobalt Oxide Nanoparticles for Tumor Theranostics. Nanoscale (2019) 11(47):23021-6. doi: 10.1039/C9NR07725A

23. Dong Y, Dong S, Wang Z, Feng L, Sun Q, Chen G, et al. Multimode ImagingGuided Photothermal/Chemodynamic Synergistic Therapy Nanoagent With a Tumor Microenvironment Responded Effect. ACS Appl Mater Interfaces (2020) 12(47):52479-91. doi: 10.1021/acsami.0c17923

24. Yang G, Xu L, Chao Y, Xu J, Sun X, Wu Y, et al. Hollow MnO(2) as a TumorMicroenvironment-Responsive Biodegradable Nano-Platform for Combination Therapy Favoring Antitumor Immune Responses. Nat Commun (2017) 8(1):902. doi: 10.1038/s41467-017-01050-0

25. Zhang S, Qian X, Zhang L, Peng W, Chen Y. Composition-Property Relationships in Multifunctional Hollow Mesoporous Carbon Nanosystems for PH-Responsive Magnetic Resonance Imaging and on-Demand Drug Release. Nanoscale (2015) 7(17):7632-43. doi: 10.1039/C5NR00451A

26. Cai X, Gao W, Ma M, Wu M, Zhang L, Zheng Y, et al. A Prussian Blue-Based Core-Shell Hollow-Structured Mesoporous Nanoparticle as a Smart Theranostic Agent With Ultrahigh pH-Responsive Longitudinal Relaxivity. Adv Mater (Deerfield Beach Fla) (2015) 27(41):6382-9. doi: 10.1002/ adma.201503381

27. Zhao H, Lv J, Li F, Zhang Z, Zhang C, Gu Z, et al. Enzymatical Biomineralization of DNA Nanoflowers Mediated by Manganese Ions for Tumor Site Activated Magnetic Resonance Imaging. Biomaterials (2021) 268:120591. doi: 10.1016/j.biomaterials.2020.120591

28. Zhou Z, Bai R, Wang Z, Bryant H, Lang L, Merkle H, et al. An AlbuminBinding $T(1)-T(2)$ Dual-Modal MRI Contrast Agents for Improved Sensitivity and Accuracy in Tumor Imaging. Bioconjugate Chem (2019) 30 (6):1821-9. doi: 10.1021/acs.bioconjchem.9b00349

29. Gomez Blanco N, Maldonado CR, Mareque-Rivas JC. Effective Photoreduction of a Pt(iv) Complex With Quantum Dots: A Feasible New Light-Induced Method of Releasing Anticancer Pt(ii) Drugselectronic Supplementary Information (ESI) Available: Details on Experimental Procedures and Additional Experimental. Chem Commun (2009) 35:5257-9. doi: 10.1039/ b910000h

30. Zhao J, Yang Y, Han X, Liang C, Liu J, Song X, et al. Redox-Sensitive Nanoscale Coordination Polymers for Drug Delivery and Cancer Theranostics. ACS Appl Mater Interfaces (2017) 9(28):23555-63. doi: 10.1021/acsami.7b07535

31. Zhang $\mathrm{M}$, Xing L, Ke $\mathrm{H}, \mathrm{He} \mathrm{YJ}$, Cui PF, Zhu Y, et al. $\mathrm{MnO}(2)$-Based Nanoplatform Serves as Drug Vehicle and MRI Contrast Agent for Cancer Theranostics. ACS Appl Mater Interfaces (2017) 9(13):11337-44. doi: 10.1021/ acsami.6b15247

32. Zhao Z, Meng H, Wang N, Donovan MJ, Fu T, You M, et al. A ControlledRelease Nanocarrier With Extracellular pH Value Driven Tumor Targeting and Translocation for Drug Delivery. Angewandte Chemie (International ed English) (2013) 52(29):7487-91. doi: 10.1002/anie.201302557

33. Hu Q, Katti PS, Gu Z. Enzyme-Responsive Nanomaterials for Controlled Drug Delivery. Nanoscale (2014) 6(21):12273-86. doi: 10.1039/C4NR04249B

34. Wang D, Lin H, Zhang G, Si Y, Yang H, Bai G, et al. Effective pH-Activated Theranostic Platform for Synchronous Magnetic Resonance Imaging Diagnosis and Chemotherapy. ACS Appl Mater Interfaces (2018) 10 (37):31114-23. doi: 10.1021/acsami.8b11408

35. Hao Y, Wang L, Zhang B, Zhao H, Niu M, Hu Y, et al. Multifunctional Nanosheets Based on Folic Acid Modified Manganese Oxide for TumorTargeting Theranostic Application. Nanotechnology (2016) 27(2):025101. doi: 10.1088/0957-4484/27/2/025101

36. Zhao Z, Wang $\mathrm{X}$, Zhang Z, Zhang $\mathrm{H}$, Liu $\mathrm{H}$, Zhu X, et al. Real-Time Monitoring of Arsenic Trioxide Release and Delivery by Activatable T(1) Imaging. ACS Nano (2015) 9(3):2749-59. doi: 10.1021/nn506640h

37. Hao Y, Wang L, Zhang B, Li D, Meng D, Shi J, et al. Manganese Dioxide Nanosheets-Based Redox/pH-Responsive Drug Delivery System for Cancer Theranostic Application. Int J Nanomedicine (2016) 11:1759-78. doi: 10.2147/ ijn.s98832

38. Wu Y, Xu L, Qian J, Shi L, Su Y, Wang Y, et al. Methotrexate-Mn(2+) Based Nanoscale Coordination Polymers as a Theranostic Nanoplatform for MRI Guided Chemotherapy. Biomaterials Sci (2020) 8(2):712-9. doi: 10.1039/ C9BM01584A

39. Fu LH, Hu YR, Qi C, He T, Jiang S, Jiang C, et al. Biodegradable ManganeseDoped Calcium Phosphate Nanotheranostics for Traceable Cascade ReactionEnhanced Anti-Tumor Therapy. ACS Nano (2019) 13(12):13985-94. doi: 10.1021/acsnano.9b05836

40. Pan YB, Wang S, He X, Tang W, Wang J, Shao A, et al. A Combination of Glioma In Vivo Imaging and In Vivo Drug Delivery by Metal-Organic Framework Based Composite Nanoparticles. J Mater Chem B (2019) 7 (48):7683-9. doi: 10.1039/С9TB01651A

41. Sun Y, Yan C, Xie J, Yan D, Hu K, Huang S, et al. High-Performance WormLike Mn-Zn Ferrite Theranostic Nanoagents and the Application on Tumor Theranostics. ACS Appl Mater Interfaces (2019) 11(33):29536-48. doi: 10.1021/acsami.9b08948

42. Yang Y, Shi H, Wang Y, Shi B, Guo L, Wu D, et al. Graphene Oxide/ Manganese Ferrite Nanohybrids for Magnetic Resonance Imaging, Photothermal Therapy and Drug Delivery. J Biomaterials Appl (2016) 30 (6):810-22. doi: 10.1177/0885328215601926

43. Yu L, Chen Y, Wu M, Cai X, Yao H, Zhang L, et al. "Manganese Extraction" Strategy Enables Tumor-Sensitive Biodegradability and Theranostics of Nanoparticles. J Am Chem Soc (2016) 138(31):9881-94. doi: 10.1021/ jacs.6b04299

44. Wang L, Li D, Hao Y, Niu M, Hu Y, Zhao H, et al. Gold Nanorod-Based Poly (Lactic-Co-Glycolic Acid) With Manganese Dioxide Core-Shell Structured Multifunctional Nanoplatform for Cancer Theranostic Applications. Int J Nanomed (2017) 12:3059-75. doi: 10.2147/IJN.S128844

45. Xi J, Da L, Yang C, Chen R, Gao L, Fan L, et al. Mn(2+)-Coordinated PDA@ DOX/PLGA Nanoparticles as a Smart Theranostic Agent for Synergistic Chemo-Photothermal Tumor Therapy. Int J Nanomed (2017) 12:3331-45. doi: $10.2147 /$ IJN.S132270

46. Bwatanglang IB, Mohammad F, Yusof NA, Abdullah J, Hussein MZ, Alitheen NB, et al. Folic Acid Targeted Mn:ZnS Quantum Dots for Theranostic Applications of Cancer Cell Imaging and Therapy. Int J Nanomed (2016) 11:413-28. doi: 10.2147/ijn.s90198

47. Song R, Zhang M, Liu Y, Cui Z, Zhang H, Tang Z, et al. A Multifunctional Nanotheranostic for the Intelligent MRI Diagnosis and Synergistic Treatment of Hypoxic Tumor. Biomaterials (2018) 175:123-33. doi: 10.1016/ j.biomaterials.2018.05.018

48. Zhu W, Liu K, Sun X, Wang X, Li Y, Cheng L, et al. Mn2+-Doped Prussian Blue Nanocubes for Bimodal Imaging and Photothermal Therapy With Enhanced Performance. ACS Appl Mater Interfaces (2015) 7(21):11575-82. doi: 10.1021/acsami.5b02510

49. Wang S, Li F, Qiao R, Hu X, Liao H, Chen L, et al. Arginine-Rich Manganese Silicate Nanobubbles as a Ferroptosis-Inducing Agent for Tumor-Targeted Theranostics. ACS Nano (2018) 12(12):12380-92. doi: 10.1021/acsnano.8b06399

50. Ryu JH, Koo H, Sun IC, Yuk SH, Choi K, Kim K, et al. Tumor-Targeting Multi-Functional Nanoparticles for Theragnosis: New Paradigm for Cancer 
Therapy. Adv Drug Deliv Rev (2012) 64(13):1447-58. doi: 10.1016/ j.addr.2012.06.012

51. Liu Y, Zhang G, Guo Q, Ma L, Jia Q, Liu L, et al. Artificially Controlled Degradable Inorganic Nanomaterial for Cancer Theranostics. Biomaterials (2017) 112:204-17. doi: 10.1016/j.biomaterials.2016.10.028

52. Ke K, Yang W, Xie X, Liu R, Wang LL, Lin WW, et al. Copper Manganese Sulfide Nanoplates: A New Two-Dimensional Theranostic Nanoplatform for MRI/MSOT Dual-Modal Imaging-Guided Photothermal Therapy in the Second Near-Infrared Window. Theranostics (2017) 7(19):4763-76. doi: 10.7150/thno. 21694

53. Sun J, Xu W, Li L, Fan B, Peng X, Qu B, et al. Ultrasmall Endogenous Biopolymer Nanoparticles for Magnetic Resonance/Photoacoustic DualModal Imaging-Guided Photothermal Therapy. Nanoscale (2018) 10 (22):10584-95. doi: 10.1039/C8NR01215F

54. Zhang H, Liu K, Li S, Xin X, Yuan S, Ma G, et al. Self-Assembled Minimalist Multifunctional Theranostic Nanoplatform for Magnetic Resonance ImagingGuided Tumor Photodynamic Therapy. ACS Nano (2018) 12(8):8266-76. doi: 10.1021/acsnano.8b03529

55. Gao S, Wang G, Qin Z, Wang X, Zhao G, Ma Q, et al. Oxygen-Generating Hybrid Nanoparticles to Enhance Fluorescent/Photoacoustic/Ultrasound Imaging Guided Tumor Photodynamic Therapy. Biomaterials (2017) 112:324-35. doi: 10.1016/j.biomaterials.2016.10.030

56. Yao Y, Li N, Zhang X, Ong'achwa Machuki J, Yang D, Yu Y, et al. DNATemplated Silver Nanocluster/Porphyrin/ $\mathrm{MnO}(2)$ Platform for Label-Free Intracellular $\mathrm{Zn}(2+)$ Imaging and Fluorescence-/Magnetic Resonance Imaging-Guided Photodynamic Therapy. ACS Appl Mater Interfaces (2019) 11(15):13991-4003. doi: 10.1021/acsami.9b01530

57. Liu H, Zhou M, Sheng Z, Chen Y, Yeh CK, Chen W, et al. Theranostic Nanosensitizers for Highly Efficient MR/fluorescence Imaging-Guided Sonodynamic Therapy of Gliomas. J Cell Mol Med (2018) 22(11):5394-405. doi: $10.1111 / \mathrm{jcmm} .13811$

58. Xu Q, Zhan G, Zhang Z, Yong T, Yang X, Gan L. Manganese Porphyrin-Based Metal-Organic Framework for Synergistic Sonodynamic Therapy and Ferroptosis in Hypoxic Tumors. Theranostics (2021) 11(4):1937-52. doi: 10.7150/thno.45511

59. He T, Qin X, Jiang C, Jiang D, Lei S, Lin J, et al. Tumor pH-Responsive Metastable-Phase Manganese Sulfide Nanotheranostics for Traceable Hydrogen Sulfide Gas Therapy Primed Chemodynamic Therapy. Theranostics (2020) 10(6):2453-62. doi: 10.7150/thno.42981

60. Yan N, Lin L, Xu C, Tian H, Chen X. A GSH-Gated DNA Nanodevice for Tumor-Specific Signal Amplification of microRNA and MR Imaging-Guided Theranostics. Small (Weinheim an der Bergstrasse Germany) (2019) 15(41): e1903016. doi: 10.1002/smll.201903016

61. Xiao J, Zhang G, Xu R, Chen H, Wang H, Tian G, et al. A pH-Responsive Platform Combining Chemodynamic Therapy With Limotherapy for Simultaneous Bioimaging and Synergistic Cancer Therapy. Biomaterials (2019) 216:119254. doi: 10.1016/j.biomaterials.2019.119254

62. Tian L, Chen Q, Yi X, Chen J, Liang C, Chao Y, et al. Albumin-Templated Manganese Dioxide Nanoparticles for Enhanced Radioisotope Therapy. Small (Weinheim an der Bergstrasse Germany) (2017) 13(25):1-9. doi: 10.1002/ smll.201700640

63. Wang Y, Wu B, Yang C, Liu M, Sum TC, Yong KT. Synthesis and Characterization of Mn:ZnSe/ZnS/ZnMnS Sandwiched QDs for Multimodal Imaging and Theranostic Applications. Small (Weinheim an der Bergstrasse Germany) (2016) 12(4):534-46. doi: 10.1002/smll.201503352

64. Gong X, Li R, Wang J, Wei J, Ma K, Liu X, et al. A Smart Theranostic Nanocapsule for Spatiotemporally Programmable Photo-Gene Therapy. Angewandte Chemie (International ed English) (2020) 59(48):21648-55. doi: $10.1002 /$ anie. 202008413

65. Liu XL, Ng CT, Chandrasekharan P, Yang HT, Zhao LY, Peng E, et al. Synthesis of Ferromagnetic Fe0.6 Mn0.4 O Nanoflowers as a New Class of Magnetic Theranostic Platform for In Vivo T1 -T2 Dual-Mode Magnetic Resonance Imaging and Magnetic Hyperthermia Therapy. Advanced Healthcare Mater (2016) 5(16):2092-104. doi: 10.1002/adhm.201600357

66. Sun H, Zhang Y, Chen S, Wang R, Chen Q, Li J, et al. Photothermal Fenton Nanocatalysts for Synergetic Cancer Therapy in the Second Near-Infrared Window. ACS Appl Mater Interfaces (2020) 12(27):30145-54. doi: 10.1021/ acsami.0c07013
67. He T, Jiang C, He J, Zhang Y, He G, Wu J, et al. Manganese-DioxideCoating-Instructed Plasmonic Modulation of Gold Nanorods for Activatable Duplex-Imaging-Guided NIR-II Photothermal-Chemodynamic Therapy. Adv Mater (Deerfield Beach Fla) (2021) 33(13):e2008540. doi: 10.1002/ adma.202008540

68. Wang H, An L, Tao C, Ling Z, Lin J, Tian Q, et al. A Smart Theranostic Platform for Photoacoustic and Magnetic Resonance Dual-Imaging-Guided Photothermal-Enhanced Chemodynamic Therapy. Nanoscale (2020) 12 (8):5139-50. doi: 10.1039/C9NR10039C

69. Hu D, Chen L, Qu Y, Peng J, Chu B, Shi K, et al. Oxygen-Generating Hybrid Polymeric Nanoparticles With Encapsulated Doxorubicin and Chlorin E6 for Trimodal Imaging-Guided Combined Chemo-Photodynamic Therapy. Theranostics (2018) 8(6):1558-74. doi: 10.7150/thno.22989

70. Li Y, Sun Y, Cao T, Su Q, Li Z, Huang M, et al. A Cation-Exchange Controlled Core-Shell MnS@Bi(2)S(3) Theranostic Platform for Multimodal Imaging Guided Radiation Therapy With Hyperthermia Boost. Nanoscale (2017) 9 (38):14364-75. doi: 10.1039/C7NR02384G

71. Jin Z, Chen D, Zhao P, Wen Y, Fan M, Zhou G, et al. Coordination-Induced Exfoliation to Monolayer Bi-Anchored $\mathrm{MnB}(2)$ Nanosheets for Multimodal Imaging-Guided Photothermal Therapy of Cancer. Theranostics (2020) 10 (4):1861-72. doi: 10.7150/thno.39715

72. Jing X, Xu Y, Liu D, Wu Y, Zhou N, Wang D, et al. Intelligent Nanoflowers: A Full Tumor Microenvironment-Responsive Multimodal Cancer Theranostic Nanoplatform. Nanoscale (2019) 11(33):15508-18. doi: 10.1039/ C9NR04768A

73. He T, Xu H, Zhang Y, Yi S, Cui R, Xing S, et al. Glucose Oxidase-Instructed Traceable Self-Oxygenation/Hyperthermia Dually Enhanced Cancer Starvation Therapy. Theranostics (2020) 10(4):1544-54. doi: 10.7150/thno.40439

74. Tang W, Fan W, Zhang W, Yang Z, Li L, Wang Z, et al. Wet/Sono-Chemical Synthesis of Enzymatic Two-Dimensional MnO(2) Nanosheets for Synergistic Catalysis-Enhanced Phototheranostics. Adv Mater (Deerfield Beach Fla) (2019) 31(19):e1900401. doi: 10.1002/adma.201900401

75. Wang Q, Wang C, Wang X, Zhang Y, Wu Y, Dong C, et al. Construction of CPs@MnO(2)-AgNPs as a Multifunctional Nanosensor for Glutathione Sensing and Cancer Theranostics. Nanoscale (2019) 11(40):18845-53. doi: 10.1039/C9NR06443E

76. Yang C, Li Y, Yang Y, Tong R, He L, Long E, et al. Multidimensional Theranostics for Tumor Fluorescence Imaging, Photoacoustic Imaging and Photothermal Treatment Based on Manganese Doped Carbon Dots. J Biomed Nanotechnol (2018) 14(9):1590-600. doi: 10.1166/jbn.2018.2565

77. Miao ZH, Wang H, Yang H, Li ZL, Zhen L, Xu CY. Intrinsically Mn2 +-Chelated Polydopamine Nanoparticles for Simultaneous Magnetic Resonance Imaging and Photothermal Ablation of Cancer Cells. ACS Appl Mater Interfaces (2015) 7(31):16946-52. doi: 10.1021/acsami.5b06265

78. Zhu Y, Wang W, Cheng J, Qu Y, Dai Y, Liu M, et al. Stimuli-Responsive Manganese Single-Atom Nanozyme for Tumor Therapy via Integrated Cascade Reactions. Angewandte Chemie (International ed English) (2021) 60 (17):9480-8. doi: 10.1002/anie.202017152

79. He M, Chen Y, Tao C, Tian Q, An L, Lin J, et al. Mn-Porphyrin-Based MetalOrganic Framework With High Longitudinal Relaxivity for Magnetic Resonance Imaging Guidance and Oxygen Self-Supplementing Photodynamic Therapy. ACS Appl Mater Interfaces (2019) 11(45):4194656. doi: 10.1021 /acsami.9b15083

80. Liu J, Du P, Liu T, Córdova Wong BJ, Wang W, Ju H, et al. A Black Phosphorus/Manganese Dioxide Nanoplatform: Oxygen Self-Supply Monitoring, Photodynamic Therapy Enhancement and Feedback. Biomaterials (2019) 192:179-88. doi: 10.1016/j.biomaterials.2018.10.018

81. Wan Q, Zou C, Hu D, Zhou J, Chen M, Tie C, et al. Imaging-Guided Focused Ultrasound-Induced Thermal and Sonodynamic Effects of Nanosonosensitizers for Synergistic Enhancement of Glioblastoma Therapy. Biomaterials Sci (2019) 7(7):3007-15. doi: 10.1039/C9BM00292H

82. Zhang R, Yan F, Chen Y. Exogenous Physical Irradiation on Titania Semiconductors: Materials Chemistry and Tumor-Specific Nanomedicine. Adv Sci (Weinh) (2018) 5(12):1801175. doi: 10.1002/advs.201801175

83. Chen J, Chen Q, Liang C, Yang Z, Zhang L, Yi X, et al. Albumin-Templated Biomineralizing Growth of Composite Nanoparticles as Smart NanoTheranostics for Enhanced Radiotherapy of Tumors. Nanoscale (2017) 9 (39):14826-35. doi: 10.1039/C7NR05316A 
84. Yang G, Zhang R, Liang C, Zhao H, Yi X, Shen S, et al. Manganese Dioxide Coated WS(2)@Fe(3) O(4)/Ssio(2) Nanocomposites for pH-Responsive MR Imaging and Oxygen-Elevated Synergetic Therapy. Small (Weinheim an der Bergstrasse Germany) (2018) 14(2):1-9. doi: 10.1002/smll.201702664

85. Song G, Liang C, Gong H, Li M, Zheng X, Cheng L, et al. Core-Shell MnSe@ Bi2 Se3 Fabricated via a Cation Exchange Method as Novel Nanotheranostics for Multimodal Imaging and Synergistic Thermoradiotherapy. Advanced Mater (Deerfield Beach Fla) (2015) 27(40):6110-7. doi: 10.1002/adma. 201503006

86. Zhou Y, Hua S, Yu J, Dong P, Liu F, Hua D. A Strategy for Effective Radioprotection by Chitosan-Based Long-Circulating Nanocarriers. J Mater Chem B (2015) 3(15):2931-4. doi: 10.1039/C5TB00063G

87. Wang M, Zhou B, Wang L, Zhou F, Smith N, Saunders D, et al. Biodegradable pHResponsive Amorphous Calcium Carbonate Nanoparticles as Immunoadjuvants for Multimodal Imaging and Enhanced Photoimmunotherapy. J Materials Chem B (2020) 8(36):8261-70. doi: 10.1039/D0TB01453B

88. Odda AH, Li H, Kumar N, Ullah N, Khan MI, Wang G, et al. Polydopamine Coated PB-MnO(2) Nanoparticles as an Oxygen Generator Nanosystem for Imaging-Guided Single-NIR-Laser Triggered Synergistic Photodynamic/ Photothermal Therapy. Bioconjugate Chem (2020) 31(5):1474-85. doi: 10.1021/acs.bioconjchem.0c00165
89. Zhang Y, Lv F, Cheng Y, Yuan Z, Yang F, Liu C, et al. Pd@Au Bimetallic Nanoplates Decorated Mesoporous $\mathrm{MnO}(2)$ for Synergistic Nucleus-Targeted NIR-II Photothermal and Hypoxia-Relieved Photodynamic Therapy. Advanced Healthcare Mater (2020) 9(2):e1901528. doi: 10.1002/adhm.201901528

Conflict of Interest: The authors declare that the research was conducted in the absence of any commercial or financial relationships that could be construed as a potential conflict of interest.

Publisher's Note: All claims expressed in this article are solely those of the authors and do not necessarily represent those of their affiliated organizations, or those of the publisher, the editors and the reviewers. Any product that may be evaluated in this article, or claim that may be made by its manufacturer, is not guaranteed or endorsed by the publisher.

Copyright $\odot 2021$ Liu and Rong. This is an open-access article distributed under the terms of the Creative Commons Attribution License (CC BY). The use, distribution or reproduction in other forums is permitted, provided the original author(s) and the copyright owner(s) are credited and that the original publication in this journal is cited, in accordance with accepted academic practice. No use, distribution or reproduction is permitted which does not comply with these terms. 